
allemande

45-2 | 2013

Images et discours de la nation

\title{
Le jeu avec les imaginaires collectifs dans les théâtres hors établissements en Allemagne
}

\section{Éliane Beaufils}

\section{(2) OpenEdition \\ 1 Journals}

Édition électronique

URL : https://journals.openedition.org/allemagne/1537

DOI : 10.4000/allemagne. 1537

ISSN : 2605-7913

Éditeur

Société d'études allemandes

\section{Édition imprimée}

Date de publication : 30 décembre 2013

Pagination : 389-402

ISSN : 0035-0974

\section{Référence électronique}

Éliane Beaufils, «Le jeu avec les imaginaires collectifs dans les théâtres hors établissements en Allemagne ", Revue d'Allemagne et des pays de langue allemande [En ligne], 45-2 | 2013, mis en ligne le 29 juillet 2019, consulté le 19 mai 2021. URL : http://journals.openedition.org/allemagne/1537 ; DOI https://doi.org/10.4000/allemagne.1537 


\title{
Le jeu avec les imaginaires collectifs dans les théâtres hors établissements en Allemagne
}

\author{
- Éliane Beaufils*
}

Les trois expériences analysées dans cet article reflètent une tendance stable voire grandissante: il s'agit de trois collectifs théâtraux qui ne se produisent pas, ou pas uniquement, dans des établissements officiels. Ils s'adressent à un public qui, moins qu'au théâtre, forme déjà une communauté. Mais ces théâtres " mineurs ", et pour cette raison moins étudiés, touchent beaucoup de monde. Qui plus est, ils s'adressent différemment au public et entendent faire venir au théâtre des gens qui n'y viendraient pas autrement. En ce sens, ce sont des théâtres qui se veulent pour tous, et qui au-delà de l'événement théâtral entendent activer un mode d'être différent en collectivité. La question est de savoir s'ils peuvent faire l'impasse sur un imaginaire national tout en contribuant à l'éveil d'une autre communauté. Si oui, quelles formes prennent cet éveil et cette communauté?

Antagon est une troupe établie depuis longtemps à Francfort. Son nom programmatique peut immédiatement être rapporté au théâtre d'action des années 1970: il importe d'activer les différends en s'émancipant de la pensée dominante et des cadres sociaux. C'est précisément l'objet du spectacle Frame Games, qui joue de manière ludique sur nos cadres de représentation. Le public est rassemblé dans la nuit estivale au bord du Main devant un espace scénique sans estrade. Derrière les tentures et les premières projections de vie urbaine dans les années 1950, il découvre des cadres, emboîtés les uns dans les autres, tous de travers mais agencés selon un ordre de grandeur décroissant. Les acteurs jouent entre les cadres écartés les uns des autres d'environ un mètre. Leur jeu sera mimétique, ludique, souvent dénué de paroles, si bien qu'ils font surgir une image après l'autre, un peu à l'instar des anciens panoptiques ou kinétoscopes. Ces images animées ont à peine le temps de se déployer avant de céder la place au prochain tableau. Comme le spectacle a lieu de nuit, les cadres constituent

* Maître de conférences, Université Paris 8. 
une forme de camera obscura, surface de projection d'images folles et évanescentes à forte dimension onirique.

Theater Anu est un autre collectif spécialisé dans les installations et les performances en plein air. Ce "théâtre poétique dans l'espace public " ${ }^{(1)}$ met souvent en place des parcours qu'il vaut mieux découvrir la nuit, en raison des nombreux jeux de lumière. Ainsi, dans le projet de 2011 Come to MoraLand, que nous avons pour notre part vu à Paderborn, les spectateurs-participants sont apostrophés par des êtres bizarres coiffés d'abat-jours et parlant le Mora, idiome que les spectateurs sont invités à apprendre afin de pouvoir mieux saluer les étrangers et communiquer avec eux. La découverte des Moraniens s'effectue au cours de rencontres avec des individus particuliers - le peintre, le musicien, le jardinier des pierres, les architectes de lumière -, le plus souvent dans des tentes qui abritent leurs travaux.

Le collectif Rimini Protokoll s'est rendu célèbre en tant que théâtre « d'experts du quotidien » ("Alltagsexperten », selon l'expression employée par les artistes eux-mêmes), parce que ses membres se fondent sur les récits et rencontres avec des « spécialistes »: ces gens sont les mieux placés pour parler du sujet puisqu'ils parlent d'eux, qu'ils transmettent leur expérience, de camionneurs (dans le spectacle Cargo Sofia, 2006), d'imams (Radio Muezzin, 2008), d'enfants (Airport Kids, 2008). De nombreux projets portent sur des étrangers et l'étrangeté, thème de leur premier succès international, où les spectateurs étaient emmenés dans une sorte de camion à paroi transparente pour « voir» en quelque sorte comme voyaient les camionneurs sillonnant l'Europe ${ }^{(2)}$. Les acteurs sont issus de milieux très différents à chaque projet, si bien que le collectif adopte presqu'au sens propre un angle différent à chaque représentation. Nous étudierons ici la pièce Black Tie, qui se réfère à une jeune Allemande d'origine coréenne, Miriam Yung Min Stein, adoptée dans les années 1970 par une famille allemande. Sa réflexion tourne autour du thème de l'identité, toujours fondée sur l'identité nationale. Il faut noter que Rimini Protokoll a eu du mal à trouver une enfant adoptée qui accepte de témoigner ${ }^{(3)}$ et que cette jeune femme a d'abord relaté son expérience dans un livre. Il s'agit donc d'un projet particulièrement travaillé et difficile.

On le voit, tant les thèmes que les cadres et modes d'action de ces théâtres sont liés à une réflexion sur le collectif, même si le collectif national n'est pas présent en tant que tel: il s'agit davantage de la "société », du pays de Mora contrastant avec l'Allemagne, et du questionnement de l'identité nationale et individuelle. Les trois groupes artistiques opposent ce faisant une forme de critique à la culture dominante (nationale). Ils reprennent dans le même temps cependant des motifs traditionnels de la culture allemande, ce qui contribue à faciliter et à enrichir la réception de leurs représentations. Last but not least, ils s'appuient sur une très forte dimension performative, qui contribue à éveiller une conscience différente de la communauté hic et nunc et permet d'intégrer tous les éléments de représentation du collectif.

1 Il se nomme lui-même ainsi: «poetisches Theater im öffentlichen Raum ».

2 Projet de Stefan Kaegi, nommé Cargo Sofia-Basel, 2006, ensuite étendu à de nombreux pays. Tous les travaux du collectif sont présentés sur internet: www.rimini-protokoll.de.

3 Propos révélé dans une discussion avec le public après la représentation à Mayence le 23 septembre 2011. 


\section{La critique des systèmes culturels nationaux et la reprise de motifs culturels fondamentaux}

Les trois théâtres veulent pratiquer un théâtre différent de celui qui est joué dans les grands établissements, jugé sclérosé et intellectuel, sclérosé parce qu'intellectuel, élitiste et exclusif. Dire que ces théâtres sont uniquement nés d'une critique du théâtre qui existe dans les « grands établissements » serait cependant réducteur. D’une certaine manière, ils conçoivent le théâtre en place comme « national », non pas comme l'avaient conçu ses fondateurs au $\mathrm{XVIII}^{\mathrm{e}}$ siècle, comme théâtre de langue et de culture allemande, mais comme système théâtral le plus cohérent et le plus riche au monde. Ils se dressent contre ce théâtre précisément parce qu'il n'est pas capable de s'adresser à toute la population, en l'occurrence de la rassembler. Amener au théâtre des gens qui n'y viendraient pas spontanément signifie aussi pour les collectifs revenir à la vocation même du théâtre, à sa dimension vivante qui, pour exister, a besoin d'espaces, de contenus, d'échanges vivants avec le spectateur afin de casser le système de communication en place pour parler en termes plus luhmanniens ${ }^{(4)}$. Leur reproche est sans doute habité par une part incontestable de populisme: il s'agit, comme le souhaitaient maintes avant-gardes de la première moitié $\mathrm{du} \mathrm{xx}^{\mathrm{e}}$ siècle, de " toucher tout le monde " et de renouer au théâtre avec la vie, aspirations qui semblent désormais naïves. Mais ils sont de ce fait mus par une volonté politique, et qui dit politique ne peut la dissocier du cadre dans lequel elle doit s'exercer - en premier lieu géographique et national.

Copyright
Theater Anu.
Photographe:
Urs Siegenthaler.

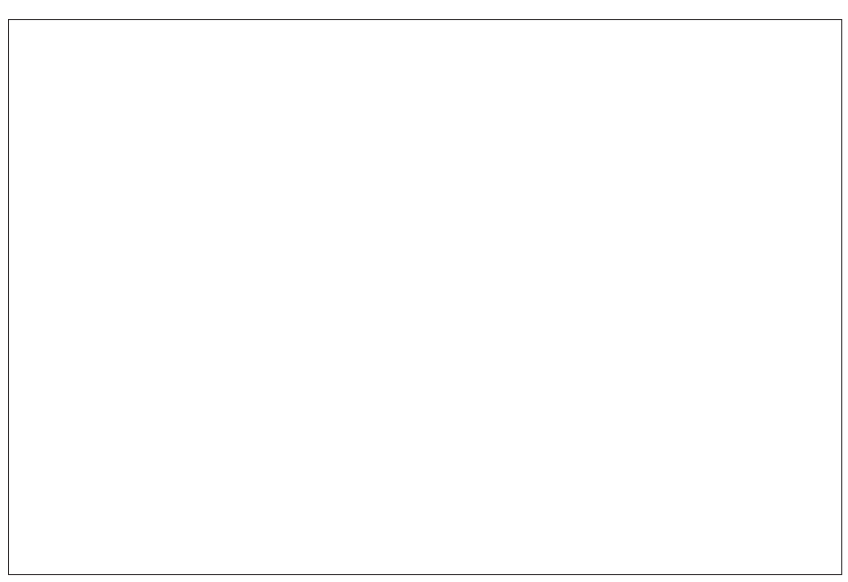

4 On rappelle que Luhmann caractérise les différents systèmes sociaux avant tout comme des systèmes de communication autopoiétiques. Voir Niklas Lummann, Soziale Systeme. Grundriss einer allgemeinen Theorie, Francfort-sur-le-Main, Suhrkamp, 1984. 
Cette critique n'est toutefois pas frontale durant les spectacles mêmes, du moins elle ne développe pas des thèmes récurrents de l'agenda médiatique et politique. Il ne s'agit pas de dénoncer, mais de montrer autre chose et de promouvoir d'autres attitudes. La seule opposition manifeste est l'opposition des lieux: ces théâtres jouent à l'extérieur, ou dans de petites salles, bref dans des lieux marginaux ${ }^{(5)}$. L'accès en est facile, les prix sont plus faibles, quand il y en a. Lors des représentations que nous avons vues, des gens venaient par hasard, en famille, entre amis. Même à Francfort, où Antagon organise depuis des années ses journées estivales, de nombreuses personnes ne connaissent pas ce festival, si bien qu'on ne peut guère parler d'un public d'habitués, où les acteurs prêcheraient à des convertis.

Cependant, "les Allemands " ne sont guère évoqués en tant que tels dans les spectacles. Si critique il y a, il s'agit davantage d'une critique de la civilisation, ou de systèmes de pensée. Frame Games reprend notamment le motif des grandes villes, souvent associé au motif du fugitif, des rencontres fugitives notamment, ainsi qu'aux transformations multiples ${ }^{(6)}$. On y voit se profiler les vocations meurtrières, sexuelles, artistiques les plus excessives, les syndromes de masse, la peur ou l'hystérie. Cette critique éminemment "moderne» se situe dans le prolongement de toute une littérature allemande, du Berlin Alexanderplatz de Döblin à l'Opéra de quat'sous brechtien, mais aussi de quantité d'auteurs étrangers du XIX ${ }^{\mathrm{e}}$ siècle, tels Eugène Sue avec les Mystères de Paris, Oscar Wilde ou Émile Zola.

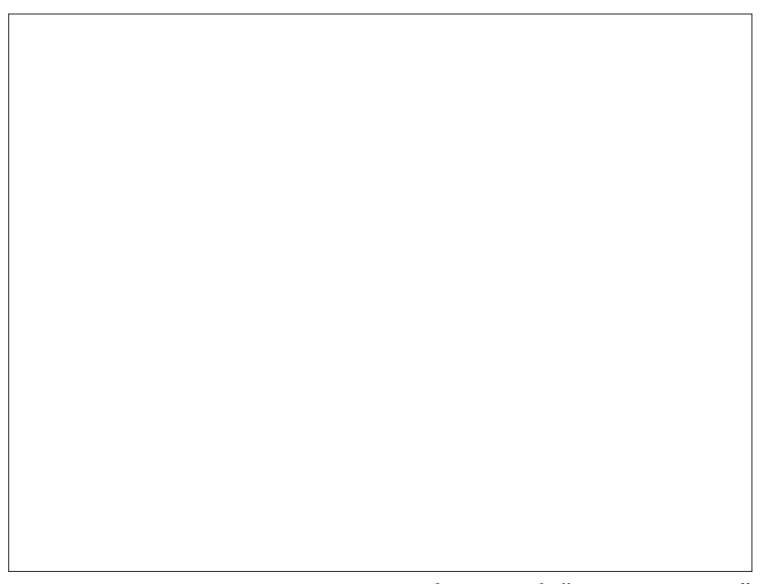

Szene aus dem Stück "Frame Games" von antagon theaterAKTion.

La critique de systèmes de pensée est plus directe, actuelle et donc acerbe chez Rimini Protokoll. En effet, Miriam dit sa souffrance à ne pouvoir percer le «trou noir de ses origines » (auquel le titre Black Tie fait allusion). Elle va jusqu'à reprocher à ses parents adoptifs une bonne conscience beaucoup trop naïve, ignorant le dilemme existentiel dans lequel ils plongent des enfants qui ne savent plus, en définitive, en quelle culture se reconnaître. Sa présentation évite néanmoins tout manichéisme. Elle revient sur l'histoire coréenne et allemande du $\mathrm{xx}^{\mathrm{e}}$ siècle, montre les paquets "Care » envoyés après la Seconde Guerre mondiale par les Américains aux Allemands meurtris et reconnaissants. Les Allemands ont constitué après-guerre une nation en développement, tributaire de l'aide étrangère, si bien qu'ils s'en sont sentis redevables et ont voulu secourir à

5 La représentation de Black Tie que nous avons vue se jouait aussi dans une institution culturelle «mineure », le KUZ à Mayence. Depuis quelques années néanmoins, le collectif est tellement renommé qu'il joue la plupart du temps dans « les grandes salles».

6 Voir à ce sujet Eva Holling, Ist alles gespielt? Blicke auf den Stadtraum im neuen Theater, Marbourg, Tectum, 2007, p. 4 sq. 
leur tour les plus démunis. Ainsi de nombreux couples allemands se sont mis, comme les Américains, à adopter les orphelins de cette autre nation détruite et divisée par la guerre qu'était la Corée. Simplement, cette présentation historique est faite par une orpheline concernée en première instance, ce qui l'investit d'une légitimité indéfectible. De plus, l'insertion dans la grande histoire généralise l'histoire singulière. Enfin, Miriam présente l'arrière-fond historique précisément comme un arrière-fond: un large panneau accueille les projections de photos retraçant l'histoire de la Corée, l'intriquant avec celle de l'Allemagne. Il reste extérieur à la jeune femme qui ne peut reprendre à son compte les bonnes raisons entendues (historiques et morales). Les petites fleurs qui tapissent le panneau sont investies d'une subtile ironie bucolique: motif joli et cher à l'enfant qu'elle était, elles représentent désormais un espace qui ne remplit pas ses promesses d'idylle. L'histoire singulière de la jeune femme confère en outre, de manière en apparence paradoxale, une teneur plus générale à la critique. Un peu comme « un Français parle aux Français ", une personne bizarre, un avatar d'Allemande parle aux Allemands, qui sont souvent aussi, surtout dans le cadre de festivals non élitistes, des Allemands aux origines diverses, des étrangers ou en septembre à Mayence, des handicapés. La parole de Miriam est comme investie intrinsèquement d'une réflexion sur nos identités composites et sur la faute qu'il y aurait à ne pas d'emblée les concevoir comme composites et relatives. Car c'est bien d'une faute dont la jeune femme accuse ses parents adoptifs, celle de ne pas avoir voulu comprendre la difficulté à être allemande dans un corps de Coréenne et plus loin, à ne pas se sentir obligée d'être reconnaissante. Miriam souligne le caractère problématique de cette reconnaissance (Dankbarkeit) qui éluderait toute vraie reconnaissance (Anerkennung), problème qui a engendré chez elle une forme d'opposition farouche à toute action caritative. On voit que les bons sentiments parentaux ont constitué une contrainte sociale abusive, manifeste également dans les sentiments excessifs qui ont habité la jeune femme: jusqu'à l'âge de onze ans, elle était de fait incapable de se séparer de sa mère, et elle a ensuite rompu tout contact avec ses parents durant ses études. Elle signifie en tout état de cause au spectateur qu'il convient de renoncer à une identité personnelle fixe, et à des valeurs inaliénables. Le thème du regard est central dans les travaux de Rimini Protokoll qui prennent Sartre presque à revers. Ce n'est pas

Rimini Protokoll:

Black Tie.

Miriam se confronte

à la culture coréenne

grâce à une jeune

femme de son âge.

Copyright

Barbara Braun. 
tant le regard de l'autre qui nous constitue, il ne devrait à vrai dire avoir aucun impact sur nous. Mais il est bon de recevoir un regard comme reconnaissance de soi. Il est donc nécessaire d'ouvrir notre regard pour ne pas enfermer les autres ni nous réduire nousmêmes. Pareille pensée peut d'ailleurs être située dans le prolongement de la culture allemande luthérienne qu'elle critique, fondée sur la vigilance à soi, dans le prolongement aussi des impératifs d'épanouissement personnel et de conscience de soi qui vont de pair avec l'ouverture à l'autre ${ }^{(7)}$. La leçon personnelle du regard et la critique exercée vis-à-vis d'une culture allemande empreinte de sentiments chrétiens, de la volonté de rendre les enfants autonomes et aimants, sont les deux faces d'une même médaille.

Ainsi, les leçons socio-psychologiques de Black Tie ont une dimension « nationale». Il est d'ailleurs révélateur que les projets de Rimini Protokoll soient tous portés par un nouveau regard sur des groupes d'individus. Qui part de groupes ou de catégories conçoit leur fort caractère structurant et, corrélativement, le blocage de notre regard par des frontières invisibles qu'il faut ouvrir. Évidemment, il convient de ne pas réduire ces projets à un contenu didactique, ils se veulent tout sauf linéaires, et ne réduisent aucunement la vision de l'Allemagne à celle des parents adoptifs ni à la culture dominante, " conductrice " (Leitkultur) ${ }^{(8)}$, qu'ils incarneraient. Le recours à des acteurs non professionnels montre précisément le caractère un peu épisodique, relatif de toute pensée dominante... dans des cadres préalables qui existent malgré tout. La critique nationale est enfin relativisée car elle passe par le filtre d'une subjectivité ostensible et que Miriam reconnaît la bonne éducation dont elle a joui.

Or les projets du théâtre Anu, bien que moins articulés et moins critiques, recouvrent également une " pédagogie nationale en creux »; mais en refusant de s'identifier au théâtre des grands metteurs en scène, il rejette aussi les principes presqu'unanimement partagés par ceux-ci : refus d'apporter des réponses, méfiance vis-à-vis de toute esthétique didactique ou de toute beauté ludique qui relèguerait l'impératif critique au second plan. Il ose ainsi plus que d'autres afficher de bonnes intentions politiques et esthétiques.

Lorsque les acteurs déguisés en Moraniens accueillent les visiteurs avec un idiome incongru en arborant le large sourire des simples, ils font appel à notre tolérance spontanée, nous invitent à adopter directement, sur leur dires, leurs gestes, leurs productions, un autre regard, nous invitent à ce même sourire des simples, à une gaieté dénuée de toute ironie mais non de cette sage allégresse qui emplit certains personnages de Hermann Hesse par exemple. Avec leur "parler étranger ", ils ne s'adressent pas simplement aux individus, mais aux individus issus d'une communauté. Il s'agit de se changer ensemble, hic et nunc, collectivité au sein de la globalité et tout d'abord au sein de la nation. La leçon est souvent assez directe: il est dit qu'il suffit de peu pour voir les choses autrement, pour se changer - adopter une attitude accueillante, se reconnaitre dans les éléments, réfléchir le monde par une multitude de miroirs qui en montrent la beauté

7 On peut interpréter cela comme un héritage du système éducatif allemand mis en place par Humboldt, mais on retombe vite dans la caricature...

8 On rappelle que le concept «Leitkultur» a été créé en 1998 par Bassam Tibi puis développé dans son livre Europa ohne Identität? Die Krise der multikulturellen Gesellschaft, Munich, Siedler, 2000. Il a donné lieu par la suite à de nombreux débats. 
mais aussi le fractionnement; il importe notamment de tout faire pour éviter ce qui est arrivé aux Moraniens. Ceux-ci ont perdu contact avec la «grande plante » qui relie tous les éléments de la nature, et cette perte, imputable à la négligence, à l'oubli de langues et de connaissances fondamentales, a amené le peuple à placer les graines restantes dans un musée, en attendant que la nature puisse à nouveau briller de mille plantes et couleurs. Le garde de cette arche de Noé botaniste nous prie donc instamment de veiller à ne pas subir la même perte et de prendre soin de nos graines ici à " Paderbé ». La déformation du nom de la ville de Paderborn montre qu'il peut s'agir de n'importe quelle ville en Allemagne. Ce qui est présenté sous forme de conte, où les personnages ont des attitudes naïves soulignant leur extravagance, aurait sans doute gagné à être présenté comme l'histoire réelle dont s'inspire le Theater Anu: le récit de l'ethnologue Heinrich Barke ${ }^{(9)}$. Le rapport à des étrangers réels aurait plus subtilement ancré ce théâtre "immersif " entre le réel et l'imaginaire, dans les possibles plus que dans le conte.

Or il suffit d'entendre cette description du voyage au pays des Moraniens, à commencer par la vertu cardinale du voyage, pour se rendre compte de son profond ancrage dans la culture romantique et idéaliste allemande. Le fait que la population soit invitée à faire des efforts dans son pays avant de voir plus loin pourrait même faire penser à Fichte, lorsqu'il voit dans les Allemands le peuple le plus apte à intégrer la raison et les valeurs universelles, un peuple élu en puissance, capable de guider les autres $^{(10)}$. Le motif romantique essentiel est bien celui du pays inaccessible, le pays de la "grande fleur» (semblable à la fleur bleue de Novalis), pays de la poésie, de l'union et de l'harmonie; il se fonde sur le lien avec mère nature, rappelle également les préceptes de Paracelse ${ }^{(11)}$; c'est le pays ou le voyage de vérité, contrée rencontrée derrière les paravents de la nôtre, grâce à l'étranger; les légendes racontées ont un rôle moteur autant que les miroirs, motif du monde caché ou renversé, les pierres, la lumière.

Antagon aussi se nourrit de représentations romantiques: il reprend le motif du rêve révélateur de vérité, de vérité supérieure, inexprimée; le jeu, dans lequel l’homme se dit, se reconnaît, non seulement révélateur, mais expression paradoxale de l'être dans l'apparence; la mise en avant de la créativité, des impressions sensuelles; le goût de la culture populaire, notamment du théâtre de foire, mêlé à des exercices circassiens (lorsque les acteurs se meuvent sur échasses).

Ces deux théâtres de l'espace public renouent en outre avec le conte. Celui-ci est vraiment conçu comme fondement culturel, on se moque du kitsch et des distinctions opérées entre culture des élites et culture populaire (sans cesse débattues ${ }^{(12)}$ ). Renouer avec la poésie populaire représente pour ces groupes une liberté d'être et de pensée, une manière d'être entière. Ils revendiquent un retour à l'essentiel, i.e. à des

9 Heinrich BARKe, Meine Reisen nach MoraLand, Berlin, Eigenverlag, 1921, p. 34.

10 Voir Fichte, $14^{e}$ discours à la nation allemande, in: Werke, Berlin, De Gruyter, 1971, p. 486-499.

11 On rappelle que pour Paracelse, le corps matériel est simplement une partie du corps cosmique. Il existe des correspondances entre le microcosme que constitue l'homme et le monde macrocosmique.

12 À commencer par les dénominations. On entend ici par «culture populaire » tout ce qui n'est pas considéré comme culture des élites, à l'instar de Jean-Paul Gabilliet, professeur à l'université Michel de Montaigne. Voir "La notion de 'culture populaire' en débat ", Revue de recherche en civilisation américaine, $\mathrm{n}^{\circ} 1$ (2009), mis en ligne le 17 juin 2009. Consulté le 14 novembre 2011, URL: http://rrca. revues.org/index173.html. 
aspirations premières de libération et d'harmonie par-delà les dysfonctionnements sociaux et à un partage de l'événement théâtral. Voilà qui rappelle les propos de Bettelheim sur les qualités des contes à « transmettre le lien social et à cultiver les vertus traditionnelles $»{ }^{(13)}$.

On pourrait rétorquer qu'Antagon et le théâtre Anu omettent de revendiquer la volonté de faire revivre une culture populaire et romantique ainsi, chez Antagon, qu'une culture moderne allemande (on pense aux motifs de la grande ville dans les années 1920). Simplement, l'opposition au théâtre des établissements publics inclut l'opposition au caractère très intellectuel de ce théâtre. Le refus de positionnement intellectuel affiché permet d'une certaine manière de se ressaisir de valeurs simples, en partie transmises par l'éducation et la formation, mais tellement présentes (par l'intermédiaire des principes éducatifs, des jeux, ou de la publicité...) qu'elles ne sont parfois plus reconnues comme romantiques ou idéalistes. Il s'agit bien néanmoins, comme le montre le nom programmatique de ces deux théâtres (antagoniste ou poétique), de défendre des valeurs et un imaginaire abandonnés par des théâtres officiels empreints de désillusion postmoderne ou à tout le moins post-adornienne.

L'idéal du théâtre populaire et le jeu avec le kitsch peuvent cependant sembler problématiques. Au « kitsch », c'est-à-dire au scénario standardisé, aux clichés, au sentimentalisme, on rapporte en général les comédies, les pièces de théâtre folkloriques, paysannes, le théâtre de foire ou les pièces politiques édificatrices ${ }^{(14)}$. On peut y rattacher en partie le théâtre Anu et Antagon. Leur intention de rassembler autour d'une culture qui renoue avec un imaginaire "allemand » et la reprise de motifs plus ou moins romantiques ne signifie rien moins qu'une construction de mythologies quotidiennes, un amalgame sans fondement réel. Comme toute culture kitsch, le théâtre Anu et Antagon entendent être divertissants, susciter une projection ou une adhésion émotionnelle, en refusant toute complexité du message: aspect ostensible quand les Moraniens parlent leur idiome et surtout quand Antagon se contente d'images scéniques sans paroles... Dans les deux cas, les spectacles ou épisodes théâtraux s'inscrivent dans un moment festif et une communauté plus larges. Ils ne sont pas sans rappeler alors le village de vacances autrichien Montafon, qui accueille chacun de façon festive et attrayante, sans qu'il ait à se poser des questions sur ses activités. Knodt caractérise celui-ci par une atmosphère débordante qui s'éprend de tout et de chacun (überflutend), si bien que les sens sont presque noyés par les sollicitations, et qu'on éprouve une facilité à être que l'auteur qualifie de « dissolution dans l'être ensemble », " conscience

13 B. Bettelheim, Psychanalyse des contes de fée, Paris, Laffont, 1977, p. 54.

14 On pourra se conférer à notre article "Kitsch oder Subversionen des Kitsches in zeitgenössischen Theaterperformances? Zum Nutzen des Weder-Noch ", à paraître prochainement dans les actes du colloque "Kitsch. Vom Nutzen der Nicht-Kunst », Kunsthochschule Halle, juin 2013. Comme on tend à recourir de plus en plus dans le théâtre contemporain à des éléments autrefois conspués comme mineurs et faciles, plusieurs livres sont parus sur le sujet ces dernières années, notamment le numéro 202 de la revue Théâtre/Public paru en 2011 sous la direction de Bernard Rothstein, « Kitsch ou néobaroque dans les scènes contemporaines " ainsi que l'ouvrage collectif paru sous la direction d'Isabelle BArbéris, Kitsch et théâtralité. Effets et affects, Dijon, Édition universitaire de Dijon, 2012. 
amalgamée avec ce qui advient " ${ }^{(15)}$. Les visiteurs n'ont plus à produire eux-mêmes les correspondances esthétiques, et fusionnent avec les autres dans les vastes machines de rythme et de lumière. On assiste en tous les cas à un « mélange de vécu personnel et collectif " ${ }^{(16)}$. Si les expériences faites avec les théâtres Anu et Antagon pouvaient être assimilées à ce genre de symbiose collective, voilà qui rendrait suspect non seulement les messages mais la communauté produite et ressentie lors du spectacle. Il n'est de fait pas possible d'éluder l'hypothèse de la régression, autrement dit: d'une recherche d'idylle illusoire, alimentée à notre bonne conscience et à des idéaux écologiques chez Theater Anu, ou nourrie d'idéologie révolutionnaire et anarchisante chez Antagon. Antagon est d'ailleurs contradictoire puisque les représentations mises en scène sont empreintes de nostalgie, de poésie, bref, sont extrêmement esthétisées plus qu'elles n'appellent à une libération. Ce réconfort par les représentations collectives pourrait répondre à un sentiment d'impuissance et de déracinement de spectateurs, comme cela fut le cas dès le XVIII ${ }^{\mathrm{e}}$ siècle lorsque le kitsch apparut et réconforta des bourgeois politiquement démunis. Le fait est qu'une « littérature, [un théâtre] qui entend en priorité divertir de larges pans de la population mais se revendique dans le même temps d'une certaine exclusivité risque de ne plus être crédible ${ }^{(17)}$. Cependant, il convient de rappeler que ces théâtres se déprennent de tout sentimentalisme, et que la réflexion, voire le questionnement sont sollicités chez Theater Anu lorsqu'il demande aux spectateurs de participer aux actions. Même lorsqu'Antagon joue avec les images et que les acteurs incarnent des stéréotypes souvent intemporels ou liés à la foire, il joue ostensiblement avec des représentations, introduisant par conséquent une distance. Il s'agit à la fois de faire revivre une ambiance festive et d'interroger l'imagerie populaire. Il faut en outre noter qu'aucune œuvre n'est jamais assurée de ne pas connaître une réception kitsch, qui la réduise à des schèmes connus ou qui nourrisse le sentimentalisme du récepteur. Mais pour être sûr que ces spectacles ne suscitent pas avant tout une effervescence régressive et illusoire, il conviendra de confronter les représentations véhiculées à la dimension performative de l'ensemble.

Rimini Protokoll ne reprend évidemment pas autant de clichés, quoiqu'on puisse retrouver dans Black Tie la notion idéaliste du voyage émancipateur lorsque Miriam parcourt le globe et retourne en Corée... pour ne pas s'y re-découvrir. Black Tie est en tous les cas une forme de théâtre documentaire. Cependant, il n'importe pas en premier lieu à Rimini Protokoll de défendre des opprimés, des laissés-pour-compte, ni de réparer les injustices et d'appeler ainsi à une action politique. Miriam a beau être issue de milieux pauvres, elle appartient désormais aux couches les plus cultivées. Journaliste, metteuse

15 «Auflösung des Subjekts im Dabeisein », " Bewusstsein amalgamiert mit Geschehen », dans Reinhard KnODT, «Liebes Montafon », in: Ästhetische Korrespondenzen. Denken im technischen Raum, Ditzingen, Reclam, 1994, p. 125 sq.

16 " durch Rhythmus und Lichtmaschinen miteinander verschmiedet ", "Vermischung von persönlichem Erleben mit Kollektiverleben ", dans R. KNODT (note 15), p. 125. Il faut noter que ce brouillage de l'individuel et du collectif constitue aussi un critère déterminant de la banalité ou trivialité pour Westerbarkey.

17 « eine Literatur, die primär breite Leserschichten unterhalten will, sich gleichzeitig jedoch exklusiv gibt, riskiert unglaubwürdig zu werden », J. GeNz, Diskurse der Wertung. Banalität, Trivialität und Kitsch, Munich, W. Fink, 2011, p. 22. 
en scène, écrivain, elle sillonne la planète. Il ne s'agit donc pas de défendre une catégorie mais de réfléchir l'existant jusque dans les bastions les plus forts de notre conscience démocratique et/ou chrétienne, telles les actions caritatives honnies par Miriam, ce qui inclut bien entendu la volonté de changer les individus et de faire prendre conscience que le monde est malléable: ce qui nous ramène par exemple à Brecht.

Ce théâtre se veut par ailleurs, comme nombre de théâtres en Allemagne, un théâtre de réflexion presque philosophique. Bien qu'il ne se fonde pas à proprement parler sur une éthique, ses sujets promeuvent une réflexion esthético-politique, prélude (comme le désirait Schiller) à un changement des mentalités, qui lui-même peut avoir des conséquences sociopolitiques. Ainsi, même s'il n'est pas autant tendu vers un but, ni animé par une intention didactique affirmée, il use quand même de moyens esthétiques pour favoriser la réflexion, qu'il s'agisse des moyens « sensuels» tels la diversité des voix et des médias, ou d'ironie, de la distance agréable et dénuée de pathos avec laquelle Miriam parle sur scène. Or user de moyens esthétiques pour favoriser la réflexion fait pour une grande partie des anciens écoliers allemands le principal intérêt du théâtre. Cet héritage est reconnu et mis en scène, notamment lorsque le collectif se produit dans de hauts lieux du théâtre comme en 2005 à Weimar dans une recréation du Wallenstein de Schiller.

Rimini Protokoll ne se contente évidemment pas d'activer chez ses spectateurs d'anciennes représentations du théâtre pour être plus percutant. Au contraire même pourrait-on dire, puisqu'il ne cesse de les surprendre au moyen d'une performativité sans cesse renouvelée, tellement effective en vérité qu'il peut se permettre en quelque sorte de renouveler le théâtre jusqu'en ses bastions et ses principaux festivals.

\section{La dimension performative des trois spectacles}

Cette dimension s'appuie au premier chef sur les lieux: anonymes et ouverts, ils peuvent rassembler les individus du monde globalisé, comme voués à penser en termes supranationaux bien plus que nationaux. Ils les rassemblent surtout en leur faisant éprouver une très grande liberté. Il se produit de la sorte un double mouvement: d'une part, les spectateurs viennent dans une sorte de no man's land, de l'autre on crée un sentiment de vivre ensemble, une expérience unique, fondant une communauté certes fragile mais réelle, puisant dans des représentations ou des «valeurs » banales ou kitsch. On plonge les spectateurs dans des cadres qui ne produisent guère de contraintes symboliques, mais en faisant appel à des représentations anciennes. Peut-on alors considérer cette manière de rassembler dans une très grande liberté comme une façon de rejouer le fondement de la communauté? Là encore, on ne peut a priori dire s'il s'agit d'un réconfort régressif ou d'une refondation.

Chez Antagon, le recours à un imaginaire un peu ancien va de pair avec une liberté de mouvements particulièrement grande. Les acteurs en effet ont beau adopter un jeu de mime soutenu par un maquillage outré, ils ne cessent d'esquisser des situations ni surtout des gestes inventifs : tomber à la diagonale l'un après l'autre relève d'une chorégraphie savamment orchestrée, de même que l'emmêlement dans de larges bandes blanches ou le jeu des mains sur les bords des cadres. L'imaginaire est hétéroclite, on voit apparaître des dauphins aussi bien que des sortes de petites filles aux allumettes, on assiste à des scènes de boucherie autant qu'à des instants de suspension poétique. Les spectateurs sont quant à eux libres d'aller et de venir comme ils l'entendent, en 
l'occurrence de manger, de boire et d'émettre des interjections... ce que la plupart oublieront de faire, mais le fait est qu'il n'existe guère de contrainte comportementale et qu'est cultivé un esprit festif qui en appelle à la curiosité de tout un chacun et qui facilite aussi les échanges après le spectacle. Parmi les effets impressionnants, il faut compter notamment le jeu sur échasses, ainsi que les épisodes de violence ou de libations. De ce fait, le groupe des spectateurs se sent spontanément lié par cet esprit de fête et les représentations sur lesquelles se fonde le spectacle muet. Les brèches sont tellement multiples qu'il est difficile de concevoir par ailleurs qu'on se projette immodérément dans ces images, qui apparaissent comme de joyeux clins d'œil, au parfum suranné, presque « vintage».

Un tel spectacle crée une « atmosphère » ${ }^{(18)}$, nourrie d'éléments passéistes autant que de jeux "pré-expressifs ", où l’on est concentré sur les métamorphoses des acteurs. Voilà qui est en particulier le cas lorsqu'ils sortent des cadres, ou en général lorsqu'ils modifient leur équilibre pour en trouver un nouveau. Comme le souligne Eugenio Barba, ce nouvel équilibre est exigeant et produit des tensions inédites. Il introduit par ailleurs une rupture de notre perception elle-même. De tels changements énergétiques aiguisent notre conscience du devenir et du présent, d'autant que les acteurs ne cessent de se costumer différemment, de se transformer. Le spectateur « se vit lui-même sur un mode particulier, comme un organisme vivant en perpétuel changement et en échange avec son environnement ${ }^{(19)}$. Comme les acteurs restent le plus souvent insérés dans leurs cadres comme des "images ", une distance est néanmoins maintenue; elle est également activée par les réminiscences du passé ou les scènes en "slow motion ». On a ainsi droit à des actions protéiformes, et à un présent instable. C'est une atmosphère auratisée plus que performative, qui soude les spectateurs en un moment de rêve à la fois commun et kaléidoscopique. Une certaine culture politique veut s'en trouver confortée et revivifiée, mais en évitant rituels, messages et structures, on lui enlève tout soubassement substantiel autre que des stéréotypes. On ne saurait donc rien déceler qui puisse structurer un imaginaire commun et encore moins une action... autre que la culture de la liberté et de la fête pour elle-même.

L'impression produite par le Theater Anu est foncièrement différente. Cela tient d'abord à ce que nous sommes participants autant que spectateurs. On entre dans le camp des Moraniens sans suivre un parcours tracé. On chemine au gré des rencontres et des discussions avec d'autres spectateurs. Le sentiment de faire face à une communauté à tous points de vue « étrangère » oblige le visiteur à se sentir étranger, en l'occurrence allemand, mais dans le même temps, il éprouve une très grande liberté. On l'invite par ailleurs à participer à des actions: danser, faire résonner l'étonnant instrument de musique qu'on entend en se bouchant les oreilles, disposer des graines et des pierres pour contribuer au développement des jardins, etc. De la sorte, le spectateur effectue un parcours initiatique malgré les réticences de sa conscience d'adulte qui y voit des enfantillages, des essais sans but ni effet rationnel. Le fait est que nous n'avons vu

18 Notion désormais courante au théâtre, d’abord définie par Gernot Böнме, Atmosphäre: Essays zu einer neuen Ästhetik, Francfort-sur-le-Main, Suhrkamp, 1995.

19 "Der Zuschauer erlebt sich in ganz besonderer Weise », " er erlebt sich als lebender Organismus, der im Austausch mit seiner Umwelt steht ", Erika Fischer-Lichte, Ästhetik des Performativen, Francfort-sur-le-Main, Suhrkamp, 2004, p. 209. 
personne quitter les Moraniens avant d'avoir goûté à tous les " ateliers ». Il se produit donc une adhésion à la découverte de l'Autre. Le spectacle fait en quelque sorte appel à la personne entière, du moins à nos trois modes de mémoire: notre mémoire sémantique, puisque les Moraniens font appel à maintes représentations, notamment romantiques et écologiques; notre mémoire épisodique, car les dessins sur sable ou les danses nous renvoient à nos propres souvenirs; et enfin notre mémoire procédurale ${ }^{(20)}$. De manière générale, plus que faire appel à notre expérience et à nos connaissances, les Moraniens entendent nous faire redécouvrir le monde. Certes, cela prend une dimension parfois très naïve, avec des images magiques ou des histoires mythologiques, et on pourrait $\mathrm{y}$ voir alors une louange « à ce qui est dépourvu d'esprit, accessible à tous " (21). Mais on découvre des qualités musicales, visuelles et charnelles d'éléments quotidiens. Redécouvrir

Rimini Protokoll: Black Tie. Miriam présente le contexte historique de son adoption. Copyright Barbara Braun. des beautés simples, cultiver une certaine naïveté, peut être très dérangeant, mais aussi très poétique, sans doute les deux si l'on en croit les réactions des spectateurs, tantôt gênées, tantôt concentrées ou souriantes. La rupture avec nos perceptions habituelles permet de se sentir intégré en fin de parcours à une communauté culturelle bizarre et indéterminée. Non pas celle, virtuelle, des Moraniens, mais celle des acteurs et spectateurs, ancrée dans l'espace ambigu qui relie la fiction, le jeu, le lieu et les réflexions sur notre civilisation.

Si l'on en vient aux conditions performatives de Black Tie, elles sont à nouveau très différentes. Nul risque ici d'auratisation ou de magie: le spectacle mise sur la relation entre le spectateur et Miriam, qui se présente comme elle est et future actrice d'ellemême. La tension issue de cette situation est à l'origine d'une présence aussi forte

20 Voir Daniel Schacter, Wir sind Erinnerung. Gedächtnis und Persönlichkeit, Reinbek, Rowohlt, 1999. Schacter définit très simplement les trois mémoires: la mémoire sémantique se rapporte au symbolique, la mémoire épisodique à des épisodes de notre vie, en l’occurrence à nos expériences personnelles, et la mémoire procédurale à l'accomplissement de gestes ou d'activités physiques connus.

21 Norbert Bolz commente l'opinion de l'artiste, pour lequel le «salut esthétique » réside dans le «quotidien, sans esprit, accessible à tous » / "ästhetisches Heil », " Alltäglichem, Geistlosen, allen Zugänglichen ", dans " Marketing als Kunst oder: was man von Jeff Koons lernen kann », in : Norbert BoLz (éd.), Riskante Bilder, Munich, W. Fink, 1996. 
qu'évidente de Miriam. Cette présence n'est pas simplement à mettre au compte de la présence de la grande histoire au sein de la petite histoire. C'est presque le contraire: comme Miriam s'attache à éviter tout pathos et même à cultiver une ironie aimable à l'égard de ses propres recherches identitaires, ironie de plus en plus difficile à mesure que cette recherche s'avère vaine, elle ne sollicite pas le spectateur, libre de se reconnaître en elle ou de s'étonner. Relation ambiguë comme ces signes que fait Miriam sans faire signe. Certes, l'arrière-fond en patchwork fonde une identité pleine de cassures et de brèches qui justifie une recherche personnelle tous azimuts. Mais le spectateur est surtout sensible à la motivation subjective et émotionnelle qui meut Miriam comme malgré elle, malgré sa distance ironique et clairvoyante. Même l'appel à la compréhension historique, par le truchement de dates et d'événements supra-individuels, par une femme qui, d'une certaine façon, en a été le jouet, nous appelle à faire le lien avec notre histoire personnelle et éveille des souvenirs de notre enfance/de notre vie au cours de ces années. Tout se passe comme si le spectateur vivait à son tour à son corps défendant le fait que les "sentiments sont les principaux moteurs de nos actions » ${ }^{(22)}$. Toujours, l'expérience de soi se mêle au message sémantique au-delà de toute représentation. Il y a co-présence de l'acteur et du spectateur, partageant moins une communauté qu'ils ne sont renvoyés à des communautés qui les dépassent et en même temps les renvoient à eux-mêmes. C'est ce sentiment de perdition et de renvoi à soi que partagent en fin de compte la somme des personnes réunies dans et par le spectacle: à travers cette interrogation de l'identité, on fait finalement l'expérience de son caractère pluriel et postsouverain ${ }^{(23)}$, qui conduit à admettre l'ambiguïté de toute valeur un peu trop affirmée. Le lieu rend en outre palpable le fondement commun si diffus qui les réunit aussi, à savoir l'appartenance à un lieu et à une langue allemands. Le sentiment de communauté se crée donc plus qu'il n'active un lien préalable. Le fondement commun, qu'on pouvait d'abord éprouver comme aléatoire ou diffus, n’a plus besoin de nous fonder en nous offrant un cadre mental fixe et réconfortant mais simplement en «étant là ». Le cadre national fait partie de l'être-là des spectateurs et de Miriam, tout pathos et toute fatalité normative lui sont enlevés.

Ces trois théâtres, hors les murs, hors lieux, dans un cadre qui active a priori le sentiment de communauté des spectateurs, renouent donc avec une motivation utopique du théâtre, alimenté à un imaginaire ancien, surtout romantique, et/ou, pour ce qui est de Rimini Protokoll, historique. Par le biais de la mémoire, de l'imaginaire, et des effets miroir avec le spectateur, voire d'une action commune avec lui, ces théâtres sont portés par la volonté de mouvoir le spectateur: volonté transformatrice qui puise dans l'émotion autant ou plus que dans l'intellect pour recréer des élans communs. Mais

22 "Vielmehr sind es Gefühle, welche die entscheidendste Motivation für unsere Handlungen liefern », E. Fischer-Lichte, Ästhetik des Performativen (note 19), p. 267.

23 Pour la notion d'identité post-souveraine, on pourra se reporter aux ouvrages de Judith Butler, ou à Philipp SCHULte, Identität als Experiment: Ich-Performanzen auf der Gegenwartsbühne, Francfortsur-le-Main, Peter Lang, 2011, qui consacre d'ailleurs un chapitre à Black Tie. On rappelle très brièvement que le terme d'identité post-souveraine est à mettre au crédit de Judith Butler: non seulement le sujet n'est plus conçu de façon essentialiste, il est donc "non-identique » à lui-même, mais la notion même de sujet relève davantage d'une fiction nécessaire, tant l'individu se construit à partir des discours ambiants. Ce terme met ainsi l'accent sur la procéduralité de l'identité. 
en se mettant en jeu, les acteurs de Theater Anu et Miriam Stein se portent également témoins d'une histoire en train de se faire, dont il faut concevoir le cadre national tout en le dépassant. Le sentiment d'une nouvelle communauté va de facto de pair avec une appréhension renouvelée de la "nation ». L'engagement critique de Miriam surtout, qui s'offre en pâture à sa réflexion et à la nôtre, permet même d'en faire un témoignage de soi apte à nous renvoyer au caractère processuel de nos identités. L'expérience d'Antagon est sans conteste la plus décevante. Vouloir faire revivre le théâtre de foire en lui donnant un cachet politique - nourrir la culture populaire comme ciment du peuple - devient kitsch, et se réduit à un artisanat esthétisant et démagogique. Mais le fait est que l'ambiance festive, les images de ville et des errements humains, ont des vertus sécurisantes et confortent peut-être les sentiments d'appartenance, au moins locale.

\section{Zusammenfassung}

Dieser Artikel stellt den Versuch dar, das Trachten dreier Theaterformen im öffentlichen Raum ernst zu nehmen. Alle drei verbindet nämlich der Wille, eine andere Art von Theater verbunden mit einem neuen Appell an das Kollektive zu entwickeln. Während Antagon das politisch-urbane Bewusstsein auf festliche Weise anspricht, bietet das Theater Anu einen partizipativen und aufklärerischen Parcours an. Rimini Protokolls Black Tie beschäftigt sich mit der Konstruktivität nationaler Identitäten in der Heranbildung der eigenen Identität. Die drei Theaterarbeiten rekurrieren auf wohlbekannte kulturelle Motive, um ein Stück (nationaler) kultureller Identität in Frage zu stellen. Antagon und das Theater Anu laufen jedoch Gefahr, den Zuschauer über wohlmeinende Klischees auf sich zurück zu werfen. Rimini Protokoll mag es eher gelingen, die kulturelle Bewusstseinsnahme zusammen mit der Befreiung bezwingender nationaler Selbstvorstellungen anzuregen.

\section{Abstract}

This article tries to take seriously the attempt of three theater forms in the public space. All three share the will to develop another kind of theater connected with a new appeal to think the collectivity. While Antagon addresses to the political-urban consciousness in festive way, the theater Anu offers the spectators a participative and educational course. Rimini Protokoll's Black Tie is busy with the constructiveness of national identities in the development of own identity. The three theater works refer to well-known cultural motives to call into question a part of (national) cultural identity. However, Antagon and the theater Anu run danger, through well-meaning clichés, to throw back the spectator on himself. Rimini Protokoll may rather succeed in stimulating the becoming conscious of cultural identity together with the liberation of defeating national selfimages. 